\title{
A NOTE ON LIFTINGS OF LINEAR CONTINUOUS FUNCTIONALS
}

\author{
HORST OSSWALD
}

(Communicated by Andreas R. Blass)

\begin{abstract}
We show that for each bounded Loeb space $\left(\Lambda, L_{\nu}(\mathfrak{A}), \hat{\nu}\right)$ a functional $\varphi \in L_{\infty}(\Lambda)^{\prime}$ has a lifting if and only if $\varphi \in L_{1}(\Lambda)$. If $p \in[1, \infty[$, then every $\varphi \in L_{p}(\Lambda)^{\prime}$ has a lifting.
\end{abstract}

\section{INTRODUCTION}

In the work of Anderson [2], Hoover and Perkins [4], Keisler [6], Lindstrøm [7], Loeb [8], and others there exists a quite long series of so-called lifting theorems, which provide a powerful tool in nonstandard analysis. Roughly speaking, they describe close connections between external and internal identities. Typical and fundamental are the following lifting theorems (1) and (2) due to Loeb [8] and Anderson [2].

Let $\left(\Lambda, L_{\nu}(\mathfrak{A}), \hat{\nu}\right)$ be the Loeb space over an internal finitely additive measure space $(\Omega, \mathfrak{A}, \nu)$, where the measure $\nu: \mathfrak{A} \rightarrow{ }^{*}[0, \infty[$ is standardly bounded.

(1) A function $f: \Lambda \rightarrow \mathbb{R}$ (then $f$ is external in general) is $L_{\nu}(\mathfrak{A})$-measurable if and only if there exists an internal $\mathfrak{A}$-measurable function $F: \Lambda \rightarrow{ }^{*} \mathbb{R}$ with * finitely many values such that $F$ and $f$ coincide up to a nullset and an infinitesimal error, i.e.,

$$
\hat{\nu}(\{f(\omega) \not \approx F(\omega)\}=0 .
$$

This internal function $F$ is then called a $\nu$-lifting of $f$.

(2) A function $f: \Lambda \rightarrow \mathbb{R}$ is $\hat{\nu}$-integrable if and only if $f$ has a $\nu$-lifting $F$, which is $S_{\nu}$-integrable, i.e., for all $H \in{ }^{*} \mathbb{N} \backslash \mathbb{N}$,

$$
\int_{\{|F| \geq H\}}|F| d \nu \approx 0 .
$$

If $F$ is an $S_{\nu}$-integrable $\nu$-lifting of $f$, then $\mathbb{E} F \approx \mathbb{E} f$, where $\mathbb{E}$ on the lefthand side denotes the expected value of $F$ with respect to $\nu$ and $\mathbb{E}$ on the right-hand side denotes the expected value of $f$ with respect to $\hat{\nu}$.

With all these lifting results in mind, perhaps one may believe that in connection with Loeb spaces liftings of separable valued functions always exist. In this note we want to characterize the nonempty class of linear continuous functionals on $L_{\infty}\left(\Lambda, L_{\nu}(\mathfrak{A}), \hat{\nu}\right)$ for which liftings do not exist. This result is

Received by the editors May 8, 1992.

1991 Mathematics Subject Classification. Primary 46B25. 
quite isolated; we do not have any applications, because the result is basically negative. In general, liftings of linear continuous functionals could be used, for example, to construct vector-valued Loeb measures and in connection with distributions.

In [10] there exist examples for linear continuous functions on nonstandard hulls of Banach spaces, which do not have liftings.

\section{LINEAR CONTINUOUS FUNCTIONALS ON $L_{p}\left(\Lambda, L_{\nu}(\mathfrak{A}), \hat{\nu}\right)$}

We work in a countably saturated nonstandard model of a standard superstructure, which contains at least the real numbers as urelements. For undefined notion and notation consult [1] or [5].

Following Loeb's work [9], the measure space $\left(\Lambda, L_{\nu}(\mathfrak{A}), \hat{\nu}\right)$ over $(\Lambda, \mathfrak{A}, \nu)$ in the introduction could be constructed as follows. A subset $N \subset \Lambda \quad(N$ may be external) is called a $\nu$-nullset if $\inf \left\{{ }^{\circ} \nu(A) \mid N \subset A \in \mathfrak{A}\right\}=0$. Fix $A \in \mathfrak{A}$ and $B \subset \Lambda$ ( $B$ may be external). Then $A$ is called a $\nu$-approximation of $B$ if the symmetric difference $A \triangle B$ of $A$ and $B$ is a $\nu$-nullset. Then it is easy to see that

$$
L_{\nu}(\mathfrak{A}):=\{B \subset \Lambda \mid B \text { has a } \nu \text {-approximation }\} \text { is a } \sigma \text {-algebra, }
$$

and that $\hat{\nu}$ is well defined and $\sigma$-additive if $\hat{\nu}(B):={ }^{\circ} \nu(A)$ for all $B \subset \Lambda$ and $\nu$-approximations $A \in \mathfrak{A}$ of $B$.

Now let $\rho: \mathfrak{A} \rightarrow{ }^{*} \mathbb{R}$ be an internal-signed finitely additive measure on $\mathfrak{A}$. By $\rho_{\perp}$ we denote the total variation of $\rho$, i.e., for all $A \in \mathfrak{A}$,

$$
\rho_{\perp}(A):=\sup \{\rho(B) \mid B \subset A \text { and } B \in \mathfrak{A}\}-\inf \{\rho(B) \mid B \subset A \text { and } B \in \mathfrak{A}\} .
$$

Assume that $\rho$ is standardly bounded. Then $\rho_{\perp}$ is also a standardly bounded finitely additive measure on $\mathfrak{A}$. Define $\hat{\rho}(B):={ }^{\circ} \rho(A)$ for all $B \in L_{\rho_{\perp}}(\mathfrak{A})$ and $\rho_{\perp}$-approximations $A \in \mathfrak{A}$ of $B$. Then $\left(\Lambda, L_{\rho_{\perp}}(\mathfrak{A}), \hat{\rho}\right)$ is a signed standard measure space.

Let $L$ be the (internal) set of all internal $\mathfrak{A}$-measurable functions $F: \Lambda \rightarrow{ }^{*} \mathbb{R}$ with * finite range. Define for all $p \in[0, \infty[$

$$
S L_{p}:=\left\{\left.F \in L|| F\right|^{p} \text { is } S_{\nu} \text {-integrable }\right\} .
$$

Then the standard part ${ }^{\circ} F$ exists $\hat{\nu}$-a.s. for each $F \in S L_{p}$. Moreover, define

$$
\begin{aligned}
L_{p} & :=\left\{f:\left.\Lambda \rightarrow \mathbb{R}|| f\right|^{p} \text { is } \hat{\nu} \text {-integrable }\right\}, \\
S L_{\infty} & :=\{F \in L|| F \mid \text { is standardly bounded }\}, \\
L_{\infty} & :=\{f: \Lambda \rightarrow \mathbb{R} \mid f \text { is essentially bounded with respect to } \hat{\nu}\} .
\end{aligned}
$$

As is the custom, two functions $f, g \in L_{p}$ are identified, if $\{\omega \mid f(\omega) \neq$ $g(\omega)\}$ is a $\hat{\nu}$-nullset. If $\mathbb{B}$ is a Banach space, by $\mathbb{B}^{\prime}$ we denote the topological dual of $\mathbb{B}$.

Fix $p \in[1, \infty]$. An internal linear function ${ }^{+} \varphi: L \rightarrow{ }^{*} \mathbb{R}$ is called a lifting of $\varphi \in L_{p}^{\prime}$, if $\varphi\left({ }^{\circ} F\right) \approx^{+} \varphi(F)$ for each $F \in S L_{p}$.

Theorem. (1) If $1 \leq p<\infty$, then each $\varphi \in L_{p}^{\prime}$ has a lifting.

(2) $\varphi \in L_{\infty}^{\prime}$ has a lifting if and only if $\varphi \in L_{1}$. (Here we identify $\varphi \in L_{1}$ with its canonical image in $L_{1}^{\prime \prime}=L_{\infty}^{\prime}$.)

Proof. (1) Let $q$ be dual to $p$, where $q=\infty$, if $p=1$. Fix $\varphi \in L_{p}^{\prime}$. There exists $g_{\varphi} \in L_{q}$ such that for each $f \in L_{p}$

$$
\varphi(f)=\mathbb{E} g_{\varphi} \cdot f .
$$


By (1) and (2), $g_{\varphi}$ has a $\nu$-lifting $G_{\varphi} \in S L_{q}$. Define for all $F \in L$

$$
{ }^{+} \varphi(F):=\mathbb{E} G_{\varphi} \cdot F .
$$

Fix $F \in S L_{p}$. By Hölder's inequality, $G_{\varphi} \cdot F$ is an $S_{\nu}$-integrable $\nu$-lifting of $g_{\varphi} \cdot f$. By theorem (2) we obtain

$$
{ }^{\circ}\left({ }^{+} \varphi(F)\right)=\mathbb{E} g_{\varphi} \cdot{ }^{\circ} F=\varphi\left({ }^{\circ} F\right) .
$$

Thus, ${ }^{+} \varphi$ is a lifting of $\varphi$.

(2) Fix $\varphi \in L_{\infty}^{\prime}$. It is well known that there exists a finitely additive measure $\tau: L_{\nu}(\mathfrak{A}) \rightarrow \mathbb{R}$ such that

$$
\tau(B)=0, \quad \text { if } \hat{\nu}(B)=0
$$

and

$$
\varphi(f)=\int f d \tau \quad \text { for all } f \in L_{\infty}
$$

(see Hewitt-Stromberg [3]). Suppose that ${ }^{+} \varphi$ is a lifting of $\varphi$, i.e.,

$$
{ }^{+} \varphi(G) \approx \varphi\left({ }^{\circ} G\right) \text { for all } G \in S L_{\infty} .
$$

Define an internal finite content $\rho: \mathfrak{A} \rightarrow{ }^{*} \mathbb{R}$ by setting $\rho(A):={ }^{+} \varphi\left(1_{A}\right)$. Fix $B \in L_{\nu}(\mathfrak{A})$. Then there exists a $\nu$-approximation $A \in \mathfrak{A}$ of $B$. Since ${ }^{+} \varphi$ is a lifting of $\varphi$, we obtain

$$
\rho(A)={ }^{+} \varphi\left(1_{A}\right) \approx \varphi\left(1_{B}\right)=\tau(B) .
$$

So if $\nu(A) \approx 0$, then $\rho_{\perp}(A) \approx 0$. Hence, by saturation,

$$
\lim _{\nu(A) \rightarrow 0}{ }^{\circ} \rho_{\perp}(A)=0 .
$$

It follows that each $\nu$-nullset is also a $\rho_{\perp}$-nullset; thus, for each $B \in L_{\nu}(\mathfrak{A})$, $\hat{\rho}(B)=\tau(B)$. Since $\hat{\rho}$ is absolutely continuous w.r.t. $\hat{\nu}$, by the RadonNikodym theorem there exists a $g \in L_{1}$ such that for each $f \in L_{\infty}$

$$
\int f d \hat{\rho}=\int f \cdot g d \hat{\nu}
$$

Therefore, for each $f \in L_{\infty}$,

$$
\varphi(f)=\int f d \tau=\int f d \hat{\rho}=\mathbb{E} f \cdot g .
$$

It follows that $g$ and $\varphi$ and be identified; thus, $\varphi \in L_{1}$.

Fix $g \in L_{1}$. Then $g$ has a $\nu$-lifting $G \in S L_{1}$. Define for all $F \in L$

$$
{ }^{+} \varphi_{G}(F):=\mathbb{E} F \cdot G .
$$

It follows that for each $F \in S L_{\infty}$, by the identification of $g$ with the functional $\varphi_{g}: L_{\infty} \rightarrow \mathbb{R}, f \mapsto \mathbb{E} f \cdot g$,

$$
{ }^{+} \varphi_{G}(F) \approx \mathbb{E}^{\circ} F \cdot g=\varphi_{g}\left({ }^{\circ} F\right) .
$$

This shows that ${ }^{+} \varphi_{G}$ is a lifting of $\varphi_{g}=g$. 


\section{REFERENCES}

1. S. Albeverlo, J. E. Fenstad, R. Høegh Krohn, and T. L. Lindstrøm, Nonstandard method in stochastic analysis and mathematical physics, Academic Press, New York, 1986.

2. R. A. Anderson, A nonstandard representation of Brownian motion and Ito-integration, Israel J. Math. 15 (1976).

3. E. Hewitt and K. Stromberg, Real and abstract analysis, Springer-Verlag, New York, 1969.

4. D. H. Hoover and E. A. Perkins, Nonstandard construction of the stochastic integral and applications to stochastic differential equations. I, II, Trans. Amer. Math. Soc. 275 (1983), $1-36,37-58$.

5. A. E. Hurd and P. A. Loeb, An introduction to nonstandard real analysis, Academic Press, New York, 1985.

6. H. J. Keisler, An infinitesimal approach to stochastic analysis, Mem. Amer. Math. Soc., no. 297, Amer. Math. Soc., Providence, RI, 1984.

7. T. L. Lindstrøm, Hyperfinite stochastic integration. I, II, III, and addendum, Math. Scand. 46 (1980).

8. P. A. Loeb, Conversion from nonstandard to standard measure spaces and applications to probability theory, Trans. Amer. Math. Soc. 211 (1975), 113-122.

9. _ـ A functional approach to nonstandard measure theory, Contemp. Math., vol. 26, Amer. Math. Soc., Providence, RI, 1984, pp. 251-261.

10. H. Osswald, Vector-valued Loeb measures and the Lewis integral, Math. Scand 68 (1991).

Mathematishes Institut, Universität MÜnChen, Theresienstr. 39, D-80333 München 2, GeRMANY 